Rapid Reviews COVID-19

\title{
Review 3: "COVID-19 and
}

its clinical severity are

associated with alterations

of plasma sphingolipids

and enzyme activities of

sphingomyelinase and

ceramidase"

Undurti Das ${ }^{1}$

${ }^{1}$ UND Life Sciences

Published on: Mar 01, 2022

License: Creative Commons Attribution 4.0 International License (CC-BY 4.0). 


\section{RR:C19 Evidence Scale rating by reviewer:}

- Potentially informative. The main claims made are not strongly justified by the methods and data, but may yield some insight. The results and conclusions of the study may resemble those from the hypothetical ideal study, but there is substantial room for doubt. Decision-makers should consider this evidence only with a thorough understanding of its weaknesses, alongside other evidence and theory. Decisionmakers should not consider this actionable, unless the weaknesses are clearly understood and there is other theory and evidence to further support it.

$* * * * * * * * * * * * * * * * * * * * * * * * * * * * * * * * * * * * * * *$

\section{Review:}

In this study, the authors found that the activities of acid and neutral sphingomyelinases (ASM, NSM), which hydrolyze sphingomyelin to ceramide, were significantly increased in COVID-19 patients. In contrast, the activity of neutral ceramidase (NC), which hydrolyzes ceramide to sphingosine, was reduced. These alterations have been proposed to result in elevated ceramide levels in SARS-CoV-2 infection. In a further extension of this study, the authors reported increased levels of ceramides 16:0 and 18:0 with the highest levels in severely affected patients and similar effects for dihydroceramide 16:0 and 18:0. In contrast, levels of (dihydro-)ceramides 24:0 were reduced. In addition, it was reported that sphingomyelin 20:0, 22:0, and 24:0 as substrates of ASM and NSM, their dihydrosphingomyelin counterparts, and sphingosine-1-phosphate were reduced in patients. These results led the authors to propose that COVID-19 is associated with a dysregulation of sphingolipid homeostasis in a severity-dependent manner. Therefore, it can be used as potential diagnostic and prognostic markers in COVID-19.

These results are certainly interesting though their value as potential markers in the diagnosis and prognosis of COVID-19 needs to be evaluated. The authors could have easily measured ceramide, sphingomyelin, and their associated enzymes in those suspected with COVID-19 and correlated the same with the severity of the disease and relevant RT-PCR test. A study as such could have given results to the value of using ceramide and sphingomyelin as potential diagnostic and prognostic markers of COVID19 as proposed. 
The authors also could have measured the expression of the number of ACE-2 receptors in the peripheral leukocytes of those with COVID-19 and correlated the same with the severity of the disease and plasma levels of ceramide and sphingomyelin and their relevant enzymes to substantiate their claims further. In addition, it would have been interesting had the authors studied the effect of ceramide and sphingomyelin on the expression and number of ACE-2 receptors in leukocytes to know whether these lipids can alter the expression of ACE-2 and, if possible, their potential influence on the affinity of ACE-2 to SARS-CoV-2 and MERS.

Suppose the results obtained indeed have any clinical value. In that case, it needs to be shown that compounds that decrease ceramide levels can decrease ACE-2 expression and alter the infectivity of SARS-CoV-2, at least in an in vitro system. In addition, it is important to show that ceramide and sphingomyelin also can alter IL-6 and TNF production and influence macrophage and $\mathrm{T}$ cell activation, at least in an in vitro study. 